\title{
The lipid bilayer at the mesoscale: a physical continuum model
}

\author{
P.L. Wilson, H. Huang, S. Takagi ${ }^{\ddagger}$
}

April 24, 2022

\begin{abstract}
We study a continuum model of the lipid bilayer based on minimizing the free energy of a mixture of water and lipid molecules. This paper extends previous work by Blom and Peletier (2004) in the following ways. (a) It formulates a more physical model of the hydrophobic effect to facilitate connections with microscale simulations. (b) It clarifies the meaning of the model parameters. (c) It outlines a method for determining parameter values so that physically-realistic bilayer density profiles can be obtained, for example for use in macroscale simulations. Points (a)-(c) suggest that the model has potential to robustly connect some micro- and macroscale levels of multiscale blood flow simulations. The mathematical modelling in point (a) is based upon a consideration of the underlying physics of intermolecular forces. The governing equations thus obtained are minimized by gradient flows via a novel numerical approach; this enables point (b). The numerical results are shown to behave physically in terms of the effect of background concentration, in contrast to the earlier model which is shown here to not display the expected behaviour. A "short-tail" approximation of the lipid molecules also gives an analytical tool which yields critical values of some parameters under certain conditions. Point (c) involves the first quantitative comparison of the numerical data with physical experimental results.
\end{abstract}

\section{Introduction}

We study the potential utility of a continuum paradigm Blom and Peletier, 2004) of the lipid bilayer as a mesoscale filter in multiscale simulations of blood flow. The two main points are first that we introduce a more physical model of the hydrophobic effect into the paradigm (for consistency with microscale simulations), and second that we investigate the role and meaning of the paradigm's

\footnotetext{
*Department of Mathematics and Statistics, University of Canterbury, Private Bag 4800, Christchurch 8140, New Zealand. Corresponding author. Email: p.wilson@math.canterbury.ac.nz

${ }^{\dagger}$ Department of Mathematics and Statistics, York University, Toronto, Canada M3J 1P3. Email: hhuang@mathstat.yorku.ca

$\ddagger$ Department of Mechanical Engineering, The University of Tokyo, Tokyo, Japan. Email: takagi@mech.t.u-tokyo.ac.jp
} 
parameters (to connect with macroscale simulations) by performing the first quantitative comparison of numerical solutions of the paradigm with physical experimental data, and in so doing provide a method for determining parameter values.

As is well-known, the cell is the fundamental element of all living matter. The activity of the cell sustains life and the cell itself is sustained by a metabolism which utilizes the mass transfer through its membrane. The cell membrane is composed of lipid molecules with proteins and other components floating in it. The dynamics of this lipid bilayer membrane become especially important in the case of dispersed components in the blood, such as red blood cells, white blood cells, platelets and so on, because the deformation dynamics of these membranes directly affect the mass transfer in the blood. These membranes are often modelled as hyperelastic due to the presence of a cytoskeleton. On the other hand, a liposome - which is composed of lipid bilayers only — is modelled as a two-dimensional fluid membrane, because the membrane lipid molecules can easily move laterally within the bilayer. Liposomes are used as drug delivery agents and artificial oxygen-carriers in blood. Nevertheless, all these cases are defined by the lipid bilayer and the behavior of the bilayer is responsible for the mass transfer through the cell membranes. Hence, the modelling of the lipid bilayer membrane from a molecular level through to the continuum level is highly anticipated to predict the mass transfer behavior in blood (Mchedlishvili and Maeda, 2001; Boryczko et al., 2003; Sugii et al., 2005; Vaidya et al., 2007).

Blood flow is a complex and major aspect of the growing field of multiscale simulations of the human body (Ayton et al., 2002; Sugii et al., 2007; Boal, 2002; Mouritsen, 2005). It is multiphase, with red blood cells (RBCs), white blood cells, platelets, artificial drug delivery agents (DDAs) and other components dispersed in a water-like plasma. It involves multiphysics, with blood components having viscoelastic properties and mutually interacting while also exchanging mass and undergoing chemical reactions. Moreover, it is multiscale; quantum mechanics at the smallest length and time scales upwards through other physics at intermediate scales, and continuum mechanics at the longest scales are all required to properly understand, predict, and control the behaviour and properties of blood. Advances in computer processing speed and storage capacity have made multiscale simulations possible, highlighting the need to understand the computational and physical interactions between the scales.

Here we focus on one crucial intermediate scale, or mesoscale, at which both molecular physics and continuum mechanics are important. This is at the membrane which surrounds RBCs and some DDAs, being typically only two molecules in thickness but extending laterally for several micrometres. Understanding how membrane composition affects deformability, and how deformation affects the mass-transfer properties of RBCs and DDAs, are key to the multiscale modelling of blood flow, since the blood vessels in which occur the greatest mass transfer from these components are also those in which they undergo the greatest deformations due to large shear forces and mechanical constriction in narrow capillaries. One significant challenge for multiscale simulations almost independent of large increases in computational power and storage, is the need to reliably transfer information from one level of the simulation to another. Presently (Young, 2001), this is mostly done ad hoc and offline, and may omit crucial mesoscale dynamics. 
The membrane is a bilayer of lipid molecules (lipids) (Mouritsen, 2005). The dipolar charge distribution in the lipid "head" groups make them able to bond with water molecules, while "tails" are indifferent to the presence of water. This amphiphilic nature leads to the hydrophobic effect which physically forms the bilayer and gives it integrity (Chandler, 2005). In this paper, we consider bilayers composed of one type of lipid.

Blom and Peletier (2004) base their continuum paradigm, herein called the "BP paradigm", on the mesoscopic dynamics framework of Fraaije (1993), minimizing a free energy for a system of lipid and water molecules. Formally, the intrinsic free energy of the system is minimized with respect to a constraint that the (unobservable) distribution of the molecules generates the (observable) continuous volume fractions, thus assuming that the microstate has relaxed to equilibrium over the relatively long time scale of the continuous description.

We introduce a new model of the inter-molecular interactions into the BP paradigm which in contrast to the original model closely represents the underlying physics of the hydrophobic effect. A further improvement is a term $\beta$ to control the decay rate of the interaction strengths. Our new model and other improvements anticipate being able to determine the values of more of the model parameters from microscale simulations (or first principles). Since tests not reported here show that both models have very similar computational costs, we concentrate largely on our new model. Our point is not that our model has computational advantages or performs better under certain conditions, but rather that it has a more physical basis and so enables a better connection to microscale simulations or first principles. It is therefore encouraging that our more physical model gives results qualitatively similar to the original model of Blom and Peletier (2004). Moreover, we show here that a key characteristic of the bilayers produced by the new model depends on a model parameter in a physically realistic way, in contrast to the original model (3.2.1)

Understanding the paradigm's parameters and the numerical roles they play is important for connecting to macroscale simulations and physical experiments. By considering the analytical origins of the paradigm, we show that some of those parameters which are at first sight physical (as opposed to purely numerical) are seen to be largely numerical, in that they cannot be directly connected a priori with physical measurements. Properties of numerical bilayers must be compared a posteriori with physical properties in order to set some parameter values. The numerical bilayers are obtained by a method of solution new to the paradigm, and are for the first time quantitatively compared with physical bilayers.

In more detail, the system of water molecules and heads and tails of lipids has a free energy split into an ideal part roughly corresponding to the Helmholtz free energy, involving only connectivity interactions, and a non-ideal part representing inter-molecular interactions. Lipid structure and configuration are therefore explicitly represented. Blom and Peletier (2004) formulate a non-ideal part of the free energy with a term reflecting the (global) compressibility of the system and another modelling the (local) hydrophobic interactions; the modelling of the hydrophobic interaction term is an open question and is not inherent to the formalism of Fraaije (1993). In this paper we introduce a new model of the hydrophobic interactions which captures the physics underlying the hydrophobic effect responsible for the formation and integrity of ordered states of amphiphiles, based on the following discussion. 
Liquid water is a dynamic hydrogen bond network in which each water molecule forms up to four hydrogen bonds with its neighbours. The non-zero dipole moment of lipid head groups makes them able to accept hydrogen bonds from water molecules (but unable to donate a bond to each other): they are hydrophilic. By contrast, the hydrophobic lipid tail groups are unable to form hydrogen bonds, although thermodynamic and electrostatic interactions between water molecules and tail groups occur, but in liquid water at room temperature the hydrogen-bond energy is typically an order of magnitude stronger than such interactions (Immergut, 1991; Mouritsen, 2005).

A cavity with a structured "surface" in the hydrogen bond network forms around a hydrophobic moeity, causing a decrease in the entropy of the system (Kronberg et al., 1995). An entropic force acts to gather together hydrophobic moieties so as to minimize the disruption to the hydrogen bond network. The physical origin of this hydrophobic effect is that water molecules close to a sufficiently large hydrophobic moiety no longer participate in four hydrogen bonds; with no attractive force towards the hydrophobic moeity, these molecules' remaining bonds now draw them away from the moiety. It is thus because lipid head groups can be nodes in the hydrogen bond network while tail groups cannot that bilayers and other structured lipid assemblies form, and this is the basis of our model.

Of the physical parameters, we take the system to be incompressible, leaving the effects of the compressibility parameter $p$ to future work. The relative tendency of heads and waters to form hydrogen bonds is modelled here by the new parameter $\gamma$ which is set as unity in this paper: the effects of $\gamma$ are also left to future work, while we simply note here that $\gamma$ moves the paradigm beyond modelling heads as attached water molecules. Herein we investigate the effects of the key physical parameters $\alpha, \epsilon, \beta, c_{0}$, and $m$. Respectively these represent temperature effects, lipid head-tail group separation distance, decay of the interaction strength, and a "background concentration" and "excess mass" of lipids (these last two terms, inherited from Blom and Peletier (2004), are clarified in this paper).

The paper is structured as follows. We introduce into the BP paradigm a new model of the inter-molecular interactions in 2.1 which forms the basis of the numerical solutions. The parameters and lipid model are discussed here. Euler-Lagrange equations, whose solution minimizes the free energy functional, are derived in $\$ 2.2$, and a novel numerical approach to solving them is given in 92.3 , along with a sample numerical result. All solutions and discussions are based on a one-dimensional model. The "smoothed" nature of the paradigm and the choice of lipid model are discussed in $\$ 2.4$.

$\$ 3$ connects the BP paradigm to physical in vitro measurements of lipid bilayers. The summary of bilayer properties in $\$ 3.1$ is used in $\$ 3.2$ to describe how numerical solutions can be calibrated to physical data. Numerical solutions are calibrated in this way for the parameters of interest in $\S 3.2 .1,2,3$. In particular, a short-range interaction (or "short-tail") approximation based on the new parameter $\beta$ is introduced in $\$ 3.2 .2$, and its effects studied analytically and numerically. This work enables guidelines on the choice of parameter values to be given in in 33.3 . The conlusions are in 4 . 


\section{The new model: derivation and numerics}

\subsection{The new model}

The original model of the hydrophobic interaction acted to move tails away from heads and waters by penalising proximity between them, mimicking the effect of the hydrophobic force but not the underlying cause, namely the uneven force distribution on water molecules in close proximity to lipid tails described above. Our approach, in direct contrast to the original model, is to promote water-water and water-head (but not head-head) proximity, modelling the hydrogen bond network, and effectively ignore the hydrophobic tails in direct imitation of the underlying physics. The relative strength of the water-water bonding preference to the water-head bonding preference is controlled by a parameter $\gamma$, meaning that heads are no longer the unphysical "attached waters" of Blom and Peletier (2004).

Formally, our system comprises "waters", each represented by a single "bead", and "lipids", each represented by a "head" bead and a "tail" bead1 connected by a rigid massless rod of length $\epsilon$. The one-dimensional model has two lipid groups aligned in the $x$-direction, normal to the bilayer plane; one group whose tails, having normalized density $u(x)$, point in the positive $x$-direction, and the other with tails of normalized density $v(x)$ pointing in the negative $x$-direction. The head beads of the first group have normalized density $\tau_{-\epsilon} u(x)=u(x+\epsilon)$, and similarly for the second. Water beads have normalized density $w(x)$. The lipids have here been chosen as the simplest allowed in the paradigm; we later (\$2.4) argue that more complex models mostly would not improve the utility of the model as a mesoscale "filter" in a multiscale simulation.

The total free energy of the system consists of three parts in the form

$$
\begin{array}{r}
E=T \int[\eta(u)+\eta(v)+\eta(w)]+\frac{p}{2} \int\left(1-u-v-\tau_{-\epsilon} u-\tau_{\epsilon} v-w\right)^{2} \\
+\alpha \int w \hat{\kappa} *\left[w+\gamma\left(\tau_{-\epsilon} u+\tau_{\epsilon} v\right)\right] .
\end{array}
$$

The new model differs from the original in the third part; the meanings of all three parts, the parameters and variables follow.

The first term, favouring spreading, represents the entropy of the system, in which $\eta(s)=s \log s$ for non-negative $s$ and $\eta(s)=\infty$ otherwise, and where $T$ is the temperature of the system. The second term is a potential energy due to compressibility, where $p$ is the system pressure.

The new form of the third term, modelling directly the underlying physics of the hydrophobic effect, involves a water-water term $w \hat{\kappa} * w$ and a water-head term $w \hat{\kappa} * \gamma\left(\tau_{-\epsilon} u+\tau_{\epsilon} v\right)$, where $*$ indicates convolution in the form

$$
(f \hat{\kappa} * g)(x)=\int f(x) \hat{\kappa}(x-y) g(y) \mathrm{d} y .
$$

The overall strength of these interactions is controlled by $\alpha$ with their relative strength controlled by $\gamma$. The interaction kernel takes the form

$$
\hat{\kappa}(s)=\kappa_{0}-\kappa(s) \text { for } \quad \kappa(s)=\delta_{\beta}(s)
$$

\footnotetext{
${ }^{1}$ All beads are of zero dimension.
} 
where $\delta_{\beta}(s)$ is a general smooth function with the properties

$$
\delta_{\beta}( \pm \infty)=0, \quad \int \delta_{\beta}=1
$$

and the constant $\kappa_{0}$ is chosen so that $\int \hat{\kappa}=1$. In this paper, we define $\kappa$ as

$$
\kappa(s)=\delta_{\beta}(s)=\frac{1}{2 \beta} e^{-\frac{|s|}{\beta}},
$$

although other choices could be considered. The new kernel $\hat{\kappa}(s)$ rewards proximity and thus represents an attractive water-water and water-head force, in contrast to the original model of Blom and Peletier (2004) which penalised watertail and head-tail proximity. The new parameter $\beta$ controls the decay of the hydrophobic interaction, and will be shown in $\$ 3.2 .2$ to introduce a straightforward analytical tool. (The notation was chosen so that the original interaction kernel of Blom and Peletier (2004) is a special case of ours, namely $\kappa(s)=\kappa_{0}-\hat{\kappa}(s)$ when $\beta=1$, but the hydrophobic integrand is quite different.)

The system is taken to be infinite with an averaged density $c_{0}$ for both $u$ and $v$. The original question addressed to the paradigm in Blom and Peletier (2004) was, in effect, whether the minimizer of the energy functional $E$ favors aggregation or spreading when an excess mass $m$ of lipids is added to the system, with all other parameters fixed. Here, we concentrate on the utility of the BP paradigm, showing that physically realistic bilayer density profiles can be obtained from it numerically. We mainly focus on the results from our new model, for the reasons given in the introduction. To simplify the analysis and computation we consider the case of periodic cells of length $2 L$. All the discussions are valid for the infinite system.

\subsection{Derivation of the Euler-Lagrange equations}

Here we take the system to be incompressible, $p=\infty$, so that

$$
1-u-v-\tau_{-\epsilon} u-\tau_{\epsilon} v-w=0 .
$$

The energy functional can be simplified as

$$
E_{I}=\int[\eta(u)+\eta(v)]+\alpha \int\left(1-u-v-\tau_{-\epsilon} u-\tau_{\epsilon} v\right) \hat{\kappa} *(1-u-v)
$$

subject to constraints

$$
\begin{gathered}
1-u-v-\tau_{-\epsilon} u-\tau_{\epsilon} v=w \geqslant 0, \\
\int\left(u+v-2 c_{0}\right)=m .
\end{gathered}
$$

The result of scaling $T$ into $\alpha$ is that we can consider the temperature effects by varying $\alpha$ (3.2.3). We have also dropped the entropy of the water molecules, which is justified since although the entropy changes of the water associated with reduced configurational arrangements around hydrophobic moieties actually assists solvation, the effect is very small (Kronberg et al., 1995). Furthermore, we have taken $\gamma=1$, effectively indicating that the electronegativities of waters and heads are equal, and leaving the effects of the relative strength of the water-water to water-head bonding preference to future work. 
Using the definition (2.5) of the interaction kernel and the mass conservation constraint (2.8b) the energy functional becomes

$$
\begin{aligned}
E_{I}=\int[\eta(u)+\eta(v)]+ & \alpha\left(1-2 c_{0}-\frac{m}{2 L}\right) \int\left(1-u-v-\tau_{-\epsilon} u-\tau_{\epsilon} v\right) \\
& -\alpha \int\left(1-u-v-\tau_{-\epsilon} u-\tau_{\epsilon} v\right) \kappa *(1-u-v),
\end{aligned}
$$

where we have chosen $\kappa_{0}=\left(2-e^{-L / \beta}\right) / 2 L$. Using the method of Lagrange multipliers we rewrite the energy functional as

$E_{T}=E_{I}+\frac{K}{2} \int \mu^{2}+\lambda_{+}\left[m-\int\left(u+v-2 c_{0}\right)\right]+\lambda_{-}\left[\int\left(u+v-2 c_{0}\right)-m\right]$,

where $K$ and $\lambda_{ \pm}$are Lagrange multipliers and $\mu=\left(u+v+\tau_{-\epsilon} u+\tau_{\epsilon} v-1\right)_{+}$, with $(\cdot)_{+}=\max \{\cdot, 0\}$.

Carrying out calculus of variations in a formal way, assuming that the order of integrations and translations can be changed wherever necessary, we derive the Euler-Lagrange equations

$$
0=\log u-\alpha \kappa *\left(2 u+2 v+2 \tau_{-\epsilon} u+\tau_{-\epsilon} v+\tau_{\epsilon} v\right)+K \mu+K \mu(x+\epsilon)+\lambda,
$$

$$
0=\log v-\alpha \kappa *\left(2 u+2 v+\tau_{-\epsilon} u+\tau_{\epsilon} u+2 \tau_{\epsilon} v\right)+K \mu+K \mu(x-\epsilon)+\lambda,
$$

where

$$
\lambda=\lambda_{-}-\lambda_{+}+1+3 \alpha-2 \alpha\left(1-2 c_{0}-m / 2 L\right) .
$$

We solve the Euler-Lagrange equations by first replacing them with evolution equations based on gradient flows:

$$
\begin{aligned}
& u_{t}=-\log u+\alpha \kappa *\left(2 u+2 v+2 \tau_{-\epsilon} u+\tau_{-\epsilon} v+\tau_{\epsilon} v\right)-K \mu-K \mu(x+\epsilon)-\lambda, \\
& v_{t}=-\log v+\alpha \kappa *\left(2 u+2 v+\tau_{-\epsilon} u+\tau_{\epsilon} u+2 \tau_{\epsilon} v\right)-K \mu-K \mu(x-\epsilon)-\lambda,
\end{aligned}
$$

for the gradients $u_{t}=-(\delta E / \delta u), v_{t}=-(\delta E / \delta v)$, with given values of $K, \lambda$. These equations are solved numerically in the next section.

There are thus nine model parameters in total: seven apparently physical and two $(K, \lambda)$ strictly numerical. $K$ and $\lambda$ will be chosen subject to stability, symmetry, and water conservation considerations described shortly. Of the physical parameters we consider herein $\epsilon, \beta, c_{0}, m, \alpha$, as described previously.

\subsection{Numerical scheme and solutions}

Our numerical scheme solves the gradient flow equations 2.13a b) to find the densities $u, v$ which minimize the Euler-Lagrange equations (2.11a, b). The approach uses a discretized grid with finite difference formulae for $u_{t}, v_{t}$. Both first- and second-order backward differencing was used, with the results in 


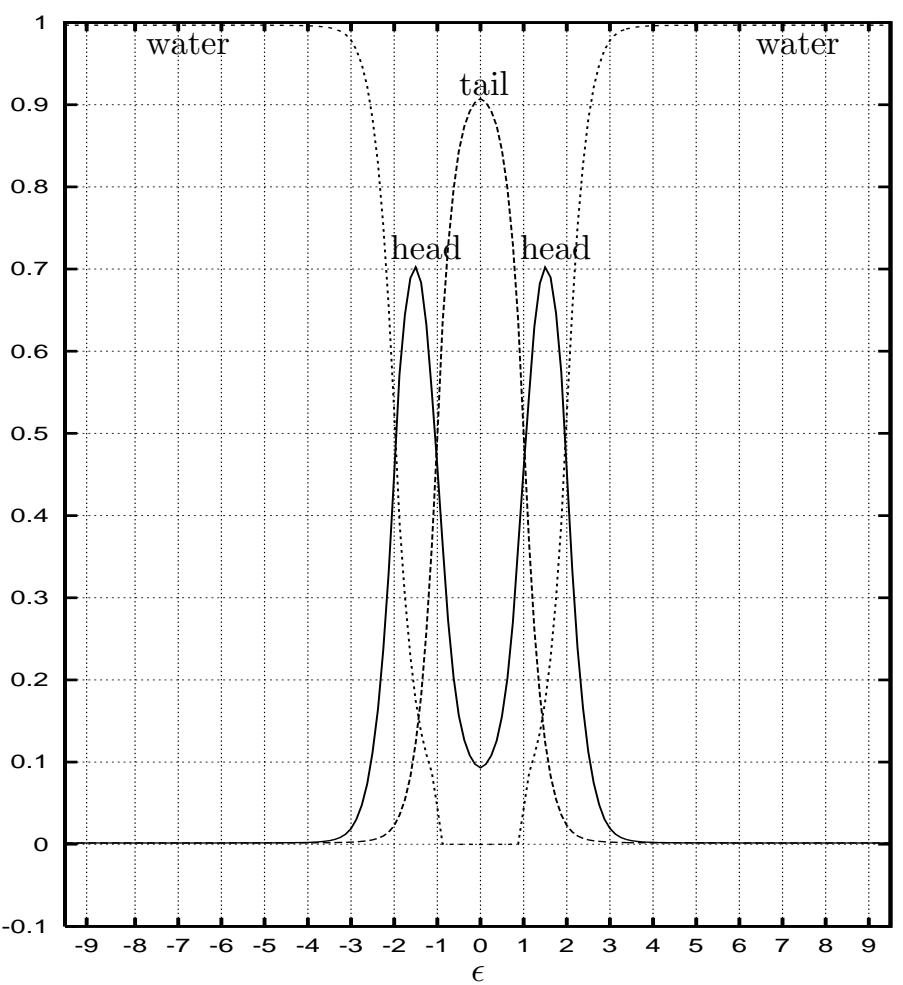

Figure 1: A sample "bilayer" for the parameter set $\alpha=3, \epsilon=2, \beta=1, c_{0}=$ $0.024, m=0.05 * 2 L$.

close agreement. Generally, very small time steps were required. The numerical domain was taken to be periodic and of length $2 L$, in contrast to Blom and Peletier (2004) who used a finite domain with small decay at the edges. In both approaches, $u, v$ can and do deviate from $c_{0}$ at the boundaries. In tests using the original model, our numerical scheme reproduced the profiles of Blom and Peletier (2004), as near as we can tell, given that their simulation conditions were not fully specified. Typical runtime on a machine with two $2 \mathrm{GHz}$ AMD Opteron 270 Dual Core procesors with 16GB of DDRS-667 SDRAM is four minutes.

The values of the Lagrange multipliers $K, \lambda$ are not specified by the model. Indeed, we require an explicit penalty term in the algorithm, effectively replacing $\lambda_{-}-\lambda_{+}$in $\lambda$ with $\lambda^{*}\left(\int\left(u+v-2 c_{0}\right)-m\right)$. Within the range of values of $K, \lambda^{*}$ for which the numerics are stable, $K$ is chosen large enough to ensure $w \geqslant 0$ but no larger, and $\lambda^{*}$ is chosen to ensure that the solutions are symmetrical, as expected. Typically, $K$ is of the order of $10^{3}$ (but can be as large as $\mathcal{O}\left(10^{4}\right)$ ), whereas $\lambda^{*}$ is around $10^{2}$.

A sample result is shown in figure 1. The system has separated into a welldefined bilayer-like profile, in which the hydrophobic tail region is separated from the water region by two peaks in the hydrophilic head group density. Although it is not our purpose to compare solutions of our model with those 
of the original model of Blom and Peletier (2004), we here note briefly that the forms are similar, but with lipids being slightly more strongly drawn into the bilayer of the new model. All the results presented in this paper are independent of domain length $2 L$ (above a certain value) and grid resolution (beyond some level of coarseness).

\subsection{Smoothing and the choice of lipid model}

Referring to our discussion in 91 of the formulation of the BP paradigm, the densities of physical lipid moieties are smoothed spatially and temporally into the densities of the model "lipids". In general, there will not be a one-to-one correspondence between each such "lipid" and the physical lipid molecules, but there is a rigorous correspondence between the densities of the one and those of the other. We prefer to call the paradigm's "lipid" a model lipid component. These components represent an as-yet unclear spatial smoothing (coarse-graining at the same length scale) and temporal smoothing of the molecular-level information. Regardless, we are free to choose any lipid component model; we have here picked the simplest. This does not mean that any information from the physical lipid is lost, but that it is smoothed into (some sum of the densities of) the two beads per component which we have. This is important from the perspective of integrating this paradigm into a multiscale simulation, for which we are interested in general bilayer characteristics such as bending rigidity which rely more on physical thickness rather than details of the bilayer profile. A simple rodand-two-beads model does the job of capturing all of the molecular data into a much smaller number of variables, and in general no more complex model lipid components need be considered. One exception might be to replace the rigid rod by a spring, since especially in the presence of embedded proteins physical lipid molecules can stretch considerably (Lee, 2003). However, even this case may be addressed by the current model lipid component.

The temporal smoothing occurs in moving from the discrete to the continuous description, working at the lipid length scale. The continuum densities are identified formally with the discrete ones over a sufficiently long time scale, but it is not clear what this time scale is. This smoothing, although unclear in detail, is central to the smoothed paradigm and is important not just because it enables access to longer time scales and hence can act as a bridge from a lower level simulation to a higher level one, but also because it captures some detail of the real thermal motion of the lipid molecules within the bilayer, which is a defining characteristic of bilayers and plays a key role in their function.

Together, the spatial and temporal smoothings are evident in the numerical results in that the model lipid components have combined to create a bilayer of total width greater than the naively-expected $2 \epsilon$, as can be seen in figure 1(see a more formal discussion in $\widehat{3.21}$.

\section{Connecting the paradigm with the physics}

\subsection{Physical bilayer properties}

Three biologically significant membrane characteristics are (1) the elastic moduli, (2) the intrinsic monolayer curvature, and (3) the bilayer thickness (Mouritsen, 


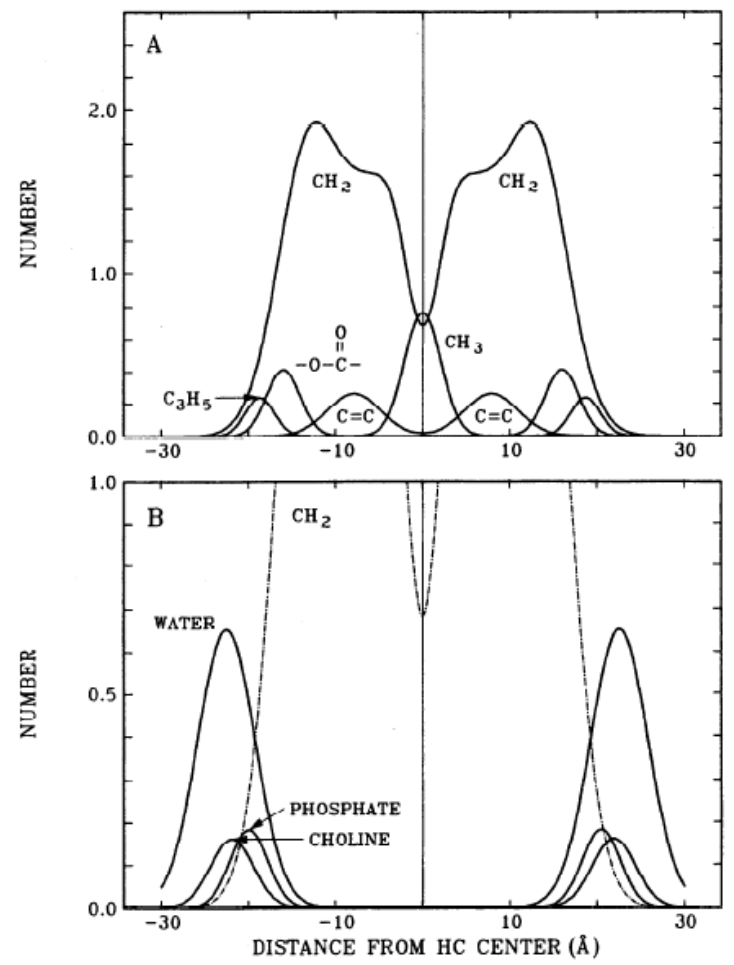

Figure 2: The experimentally-determined structure of a DOPC bilayer (figure reproduced from Wiener and White (1992) with kind permission of the authors and the Biophysical Society.)

2005). The latter is the focus of the present section ((1) and (2) require working in higher dimensions).

The averaged thickness $d_{\mathrm{SZ}}$ of the hydrophobic core, or saturation zone, is a common physical measure of bilayer thickness. In a physical system this can be increased by the following means (Mouritsen, 2005, §8.3): increasing the length $l_{\mathrm{T}}$ of the tails; replacing the double carbon bonds by single bonds in the tails; decreasing the degree of hydration; increasing the cholesterol concentration; decreasing the temperature. (The latter means each lipid has less kinetic energy, making all lipids on average less convoluted, and hence longer on average, while in a bulk effect lowering the temperature makes the arrangement of lipids more crystalline. Both effects increase $d_{\mathrm{SZ}}$.) These effects can increase $d_{\mathrm{SZ}}$ by several percent (Mouritsen, 2005, §9.2).

The main approaches used in physical experiments to determine the timeaveraged structure of lipid bilayers, and hence their thickness, exploit the high structural periodicity in the direction normal to the bilayer, for example in combining diffraction data from x-ray and neutron scattering (Wiener and White, 1992). One such data set is represented in figure 2 for the DOPC lipid molecule. In this figure, the water density measures only the waters of hydration (those bonded to head groups). 


\subsection{Making comparisons with physical meaurements}

For our analysis the key features of figure 2 are the saturation zone, defined as that region where $w \approx 0$, the density curves of the end of the tail (here, the $\mathrm{CH}_{3}$ moiety of DOPC), and those of the head group (here, choline and phosphate). The DOPC bilayer will form the basis of the remaining work in this paper, but the key point is that any single-species bilayer can be simulated by the BP paradigm, when basic structural details are known from experiments.

We consider only those numerical results with a clear bilayer structure like that shown in figure 1, namely with a single saturation zone of width $d_{\mathrm{SZ}}$, a single tail peak with exactly two transitions from concavity to convexity, and two head peaks of equal width $d_{\mathrm{HZ}}$, each likewise with exactly two transitions from concavity to convexity. Macro-separation of the two species $u$ and $v$ can and does occur without these criteria being met, but our analysis is here concerned only with those profiles rigourously of this form. The head zone width $d_{\mathrm{Hz}}$ is defined in the following way. Using the left-hand head peak, let $x_{L}, x_{R}$ be points immediately to the left and right of the peak satisfying $h_{x}=0, h_{x x}>0$, i.e. local minima, with the local maxima of the head peak located at $x^{*}$. We then find $x_{1}, x_{2}$ from $h\left(x_{1,2}\right)=\frac{1}{2}\left(h\left(x_{L, R}\right)+h\left(x^{*}\right)\right)$ and define $d_{\mathrm{HZ}}=x_{2}-x_{1}$. Defining the head zone width in this way as running from the left-hand midpoint to the right-hand midpoint of the peak is not unusual (e.g. Mouritsen (2005, fig 8.1)), but none of our conclusions is changed significantly by defining it as $x_{R}-x_{L}$.

Lipid bilayers have different $d_{\mathrm{HZ}}$ and $d_{\mathrm{SZ}}$ depending on the lipids of which they are composed. Specifically, the ratio $d_{\mathrm{HZ}} / d_{\mathrm{SZ}}$ depends on the choice of lipid molecule (other factors such as temperature being equal) and so characterizes the bilayer properties for our purposes. For the DOPC bilayer of figure 2 , $d_{\mathrm{HZ}} / d_{\mathrm{SZ}} \approx 0.4$.

We now show that varying the key paradigm parameters of $\$ 2.1$ enables a solution to be found corresponding closely to any desired physical bilayer, with the DOPC bilayer as our example. The method of selecting the values of the parameters is summarized in $\$ 3.3$.

\subsection{1 $c_{0}, m$ and the critical micelle concentration}

Blom and Peletier (2004) called $c_{0}$ the "background concentration" and $m$ the "excess lipids" above the background concentration. This terminology stems from the physical phenomenon known as the critical micelle concentration (CMC), in which the monomer density of lipids in solution only increases up to the CMC, beyond which the excess lipids aggregate into ordered structures. The type of ordered structure depends on the lipid geometry (Boal, 2002). The BP paradigm takes $c_{0}$ to be the background concentration to which an excess quantity of lipids $m$ is added.

When implementing the paradigm numerically we face the problem that the smoothing of $\$ 2.4$ only reveals the lipid species a posteriori once the numerical profiles are compared with experimental ones. Without knowing the species, and without also knowing a priori the temperature of the system, the CMC cannot be known at the start of the simulation, and hence neither can $c_{0}$ and $m$. Moreover, the CMC refers to an average lipid density, which here would be

$$
\bar{\rho}=\frac{2}{2 L} \int u+v=\frac{m}{L}+4 c_{0} .
$$




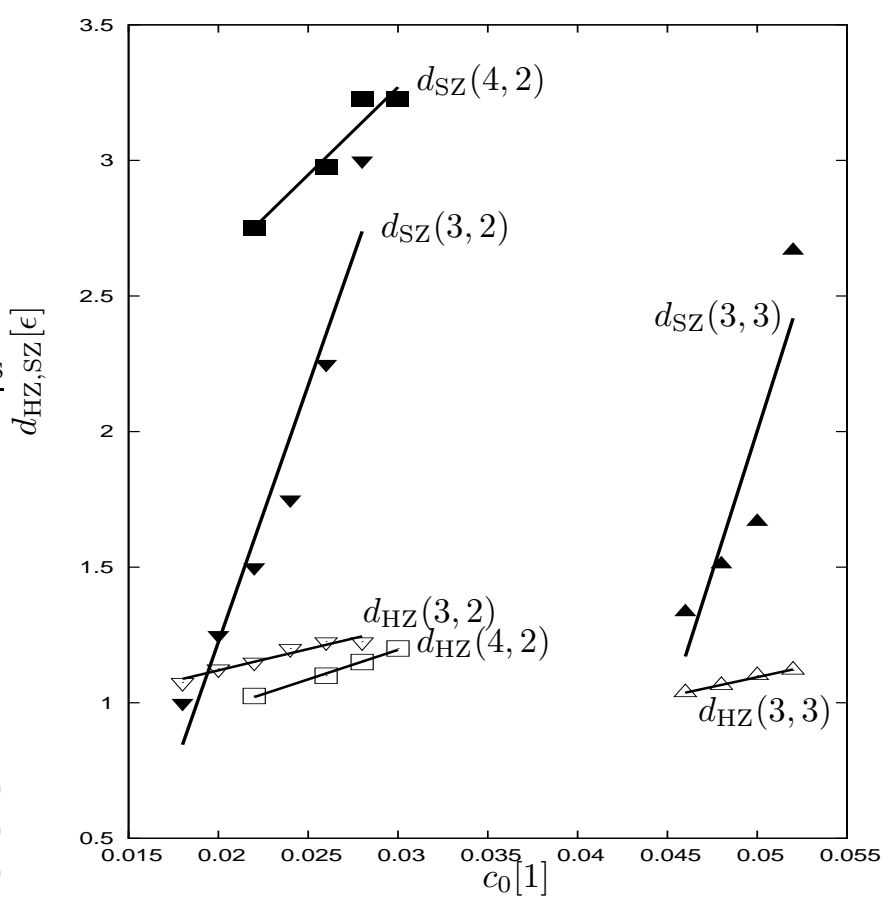

Figure 3: $d_{\mathrm{HZ}}$ and $d_{\mathrm{SZ}}$ against $c_{0}$ for three different pairs $(\alpha, \epsilon)$, with all other parameters equal. Straight lines of best fit have been drawn.

Consequently, the true background density is $m / L+4 c_{0}^{*}$, where $c_{0}^{*}$ is the value of $c_{0}$ for which aggregates first form (with all other parameters fixed), and the total excess of lipids is $8 L\left(c_{0}-c_{0}^{*}\right)$. Since $m$ and $c_{0}$ combine to form the CMC, we can numerically fix $m$ at a working value and vary $c_{0}$ only.

We note here that the above argument suggests that $u$ and $v$ should decay to $\left(m / L+4 c_{0}^{*}\right) / 4$ far from the bilayer, but both the periodic domain used in the present numerical simulations, and the finite arbitrary value of $L$ used in the numerical method of Blom and Peletier (2004), allow $u$ and $v$ to vary away from the background concentration at the "edges".

In figures 3 and 4 we consider three data sets in which the parameters $\alpha, \epsilon$, and $c_{0}$ vary, the values of $\alpha$ and $\epsilon$ being given in parentheses on the graphs. The widths $d_{\mathrm{HZ}}$ and $d_{\mathrm{SZ}}$ are normalized on $\epsilon$ in these figures. Numerical data is represented by points and a straight line of best fit is drawn in each case.

In the numerical system, as $c_{0}$ increases beyond $c_{0}^{*}$, the excess lipids thus supplied should be drawn into the bilayer with the numerical background concentration remaining more or less the same. This required increase of $d_{\mathrm{Hz}}$ and $d_{\mathrm{SZ}}$ with increasing $c_{0}$ can be seen in figure 3. Although this is encouraging, this one-dimensional study cannot yet reveal the true physicality of the models in terms of varying $c_{0}$. In a physical system, as more lipids are added to the solution beyond the CMC, more micelles (or other ordered structures) are formed, with the average size of each barely affected (Boal, 2002). Such a test must wait for two-dimensional results.

More importantly, the data can be combined into the ratio $d_{\mathrm{HZ}} / d_{\mathrm{SZ}}$ as 


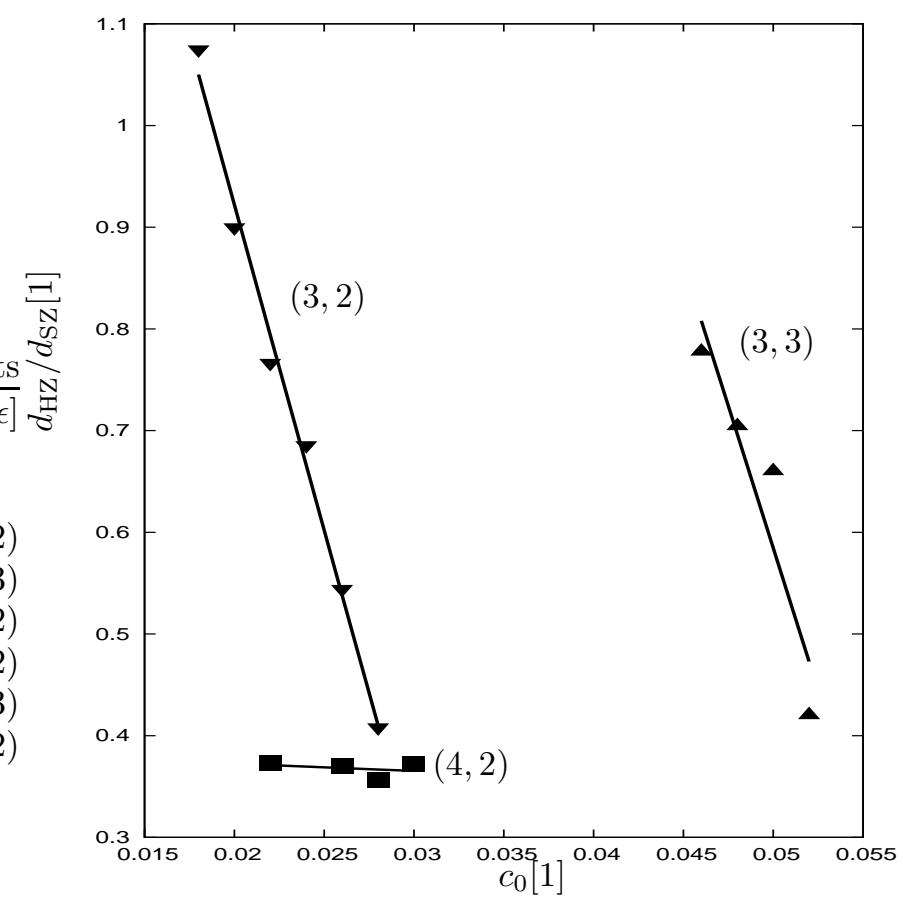

Figure 4: The ratio $d_{\mathrm{Hz}} / d_{\mathrm{SZ}}$ against $c_{0}$ for the three parameter sets of figure 3 , Straight lines of best fit have been drawn.

shown in figure 4. Taking the example of a DOPC bilayer, the data shows that we can choose suitable parameter sets such that $d_{\mathrm{HZ}} / d_{\mathrm{SZ}} \approx 0.4$, namely $\left(\alpha, \epsilon, c_{0}\right)=(3,2,0.028)$ for which $d_{\mathrm{HZ}} / d_{\mathrm{SZ}}=0.41,\left(\alpha, \epsilon, c_{0}\right)=(4,2,0.022)$ for which $d_{\mathrm{HZ}} / d_{\mathrm{SZ}}=0.37$, and $\left(\alpha, \epsilon, c_{0}\right)=(3,3,0.052)$ for which $d_{\mathrm{HZ}} / d_{\mathrm{SZ}}=0.42$. We expect that ultimately $\alpha$ can be set physically (see also 3.2 .3 ) and that the role of $\epsilon$ is more one of clarifying the structure (\$3.2.2), so that effectively here our only choice would be $c_{0}$, and the data shows that a value can be chosen which yields a bilayer characteristic close to the desired one. This is expected to hold for lipids other than DOPC since there was nothing special about our choice of this molecule.

Although it is not our intention to directly compare the new model with the original, it is worth noting here that the original model does not show the expected increase of $d_{\mathrm{HZ}}$ with $c_{0}$ : indeed, an inverse relationship between $d_{\mathrm{HZ}}$ and $c_{0}$ holds for numerical data generated by the original model, as shown in figure 5

\subsection{2 $\epsilon$ and the interaction decay length}

That $\epsilon$ is not an actual lipid length has already been discussed. What role, then, does it play?

Varying $\epsilon$ changes the degree of separation of the head and tail regions, larger $\epsilon$ giving clearer bilayer structure. With our definition (p. 111) of what constitutes a realistic bilayer profile, we need only consider a few order unity values of $\epsilon$; 


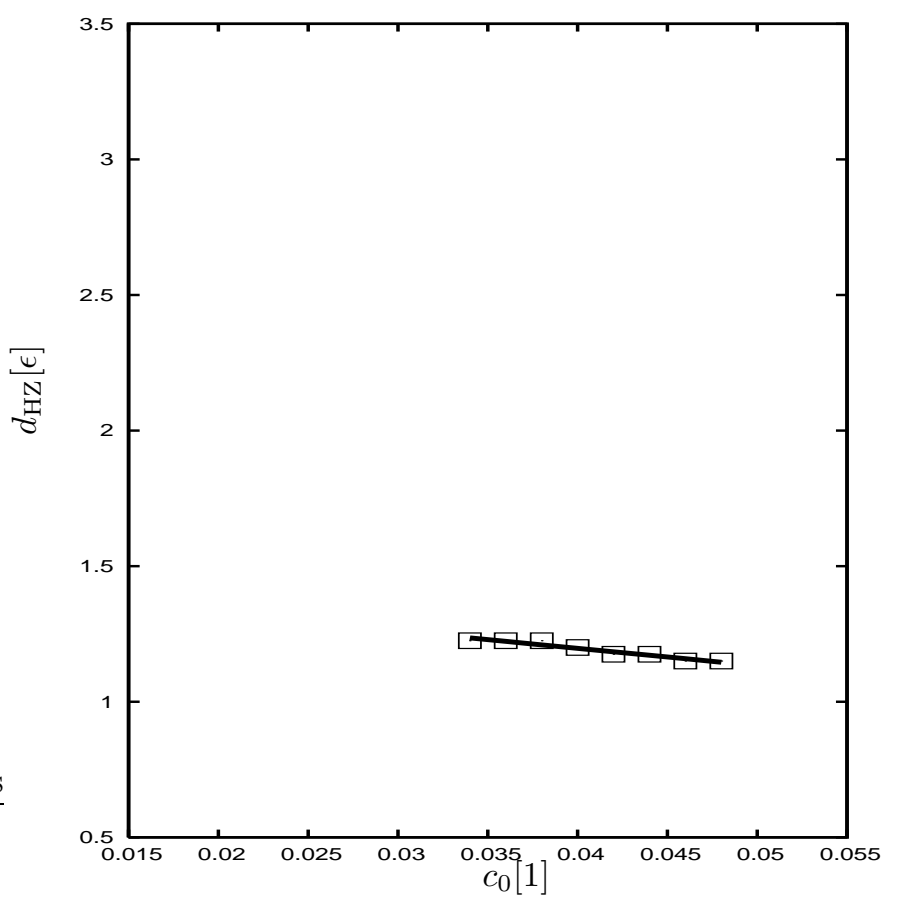

Figure 5: Example data for the original model: $d_{\mathrm{Hz}}$ against $c_{0}$ for $(\alpha, \epsilon)=(4,2)$, plotted on the same scale as figure 3. A straight line of best fit has been drawn, indicating a non-physical inverse relationship between $d_{\mathrm{HZ}}$ and $c_{0}$.

see $₫ 3.3$

Turning to the parameter $\beta$, which controls the interaction decay length, we consider another special case of the kernel function of equation (2.5). Our "short-range interaction" approximation takes $\beta \rightarrow 0$, so that the kernel function approaches the delta function

$$
\kappa(s)= \begin{cases}1, & s=0 ; \\ 0, & s \neq 0 .\end{cases}
$$

The Euler-Lagrange equations (2.11) then become

$$
\begin{aligned}
& 0=\log u-\alpha\left(2 u+2 v+2 \tau_{-\epsilon} u+\tau_{-\epsilon} v+\tau_{\epsilon} v\right)+K \mu+K \mu(x+\epsilon)+\lambda, \\
& 0=\log v-\alpha\left(2 u+2 v+\tau_{-\epsilon} u+\tau_{\epsilon} u+2 \tau_{\epsilon} v\right)+K \mu+K \mu(x-\epsilon)+\lambda,
\end{aligned}
$$

which in the case of $K \rightarrow \infty$ reduce to

$$
\begin{aligned}
& \log u-\alpha\left(2 u+2 v+2 \tau_{-\epsilon} u+\tau_{\epsilon} v+\tau_{\epsilon} v\right)=-\lambda, \\
& \log v-\alpha\left(2 u+2 v+\tau_{-\epsilon} u+\tau_{\epsilon} u+2 \tau_{\epsilon} v\right)=-\lambda .
\end{aligned}
$$

Separating $\lambda$ into a constant term plus a term dependent on $c$ by rewriting (2.12) as $\lambda=\bar{\lambda}+2 \alpha\left(2 c_{0}+m / 2 L\right)$, we look for constant solutions $u=v=c$, obtaining

$$
\log c-4 \alpha c=-\bar{\lambda} .
$$



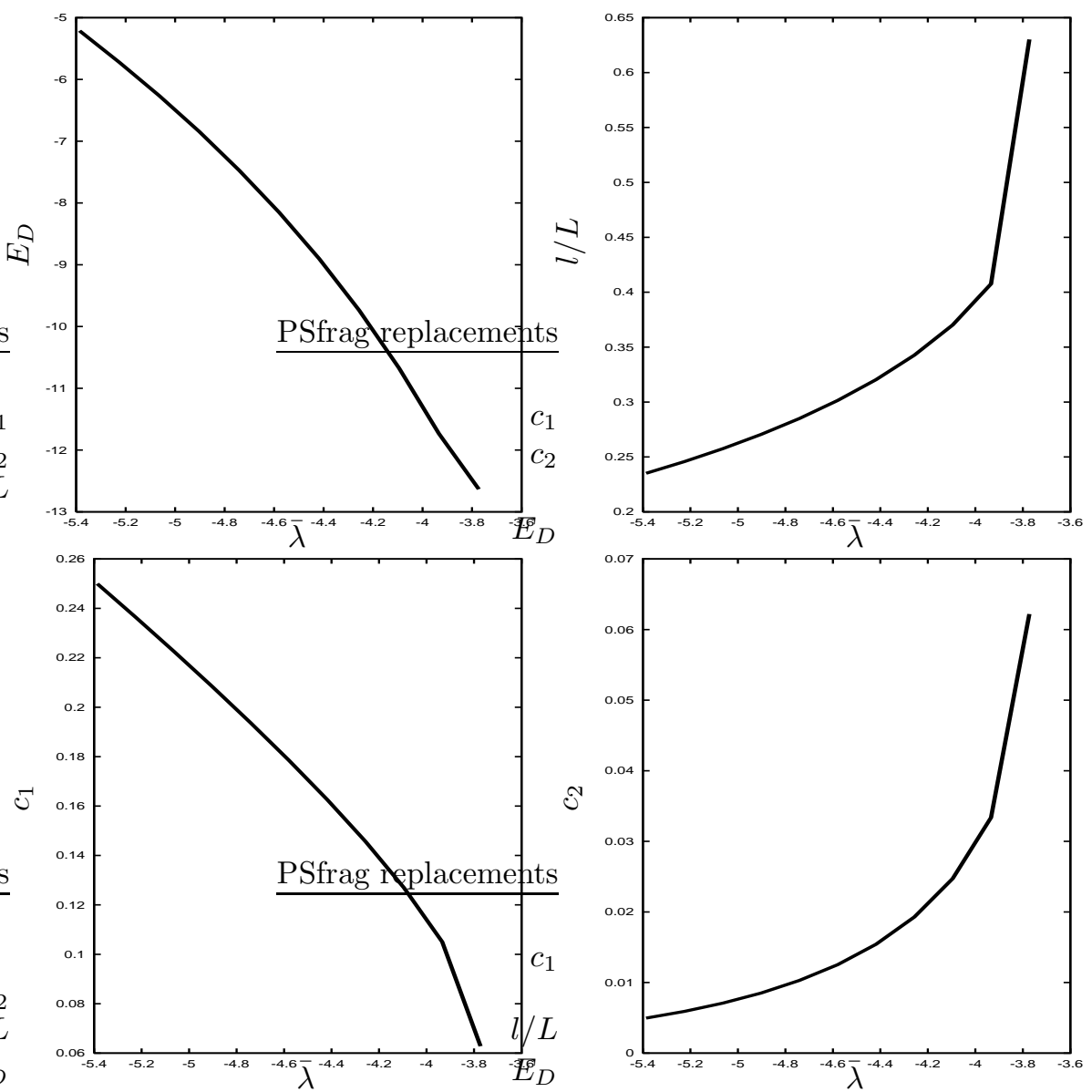

Figure 6: Solutions of equations 3.123.113.5 for the parameters $\alpha=4, \epsilon=$ $2, c_{0}=1 /(4 \alpha), m=c_{0} / 10, L=20$.

Differentiating (3.5) with respect to $c$ yields the critical concentration $c_{c}$ as the first necessary condition for the existence of a solution:

$$
c=c_{c} \equiv \frac{1}{4 \alpha} \text {. }
$$

The short-range interaction tool thus gives a simpler way to find the same result (3.6) as Blom and Peletier (2004). To simplify our discussion, we assume that $c_{0}=c_{c}=1 / 4 \alpha$ and take $\alpha \geqslant 1$.

The other necessary condition for the existence of a solution is estimated as follows. From the incompressibility condition (2.8a) we see that $c \leqslant 0.5$. Substituting $c=0.5$ into (3.5) gives

$$
\bar{\lambda}_{\min }=-\log 2-2 \alpha .
$$

When there is phase separation, (3.5) must have more than one solution: indeed, $\bar{\lambda}$ must also be less than $\bar{\lambda}_{\max }$ where

$$
\bar{\lambda}_{\max }=\log c_{c}-4 \alpha c_{c}=-\log (4 \alpha)-1,
$$


in which case there is only one solution. When $\beta=0$ the incompressibility condition implies $c \leqslant 0.25$, and therefore

$$
\bar{\lambda}_{\min }=-\log 4-\alpha .
$$

With $\bar{\lambda}_{\min }<\bar{\lambda}<\bar{\lambda}_{\max }$ there are many possible states. The relevant question is whether there exists a global minimum of the $\delta$-function free energy

$E_{D}=\lim _{\beta \rightarrow 0} E_{I}=\int \eta(u)+\eta(v)+\alpha\left(u+v-2 c_{0}-\frac{m}{L}\right)\left(1-u-v-\tau_{-\epsilon} u-\tau_{\epsilon} v\right)$.

With $\bar{\lambda}$ chosen between its minimum and maximum values, we assume that $u=v=c_{1}$ for $-l<x<l$ and $u=v=c_{2}$ elsewhere, where $c_{1}$ and $c_{2}$ are two distinct solutions of (3.5). We also assume that the solution is periodic with period $2 L$. The mass conservation (2.8b) gives

$$
l=\frac{m+4 L\left(c_{0}-c_{2}\right)}{4\left(c_{1}-c_{2}\right)},
$$

which in (3.10) yields

$$
\begin{aligned}
\frac{E_{D}}{4}= & l\left[c_{1} \log c_{1}-c_{2} \log c_{2}+2 \alpha\left(2\left(c_{1}+c_{2}\right)+2 c_{0}+\frac{m}{L}\right)\left(c_{1}-c_{2}\right)\right] \\
& +L\left[c_{2} \log c_{2}+\alpha\left(c_{0}+\frac{m}{2 L}\right)\left(4 c_{2}-1\right)+4 \alpha c_{2}^{2}\right]-\epsilon \alpha\left(c_{1}-c_{2}\right)^{2} .
\end{aligned}
$$

In figure 6. we have plotted $E_{D}$ as a function of $\bar{\lambda}$, for $(\alpha, \epsilon)=(4,2)$. We can see that the global minimum occurs when $\bar{\lambda}=\bar{\lambda}_{\max }$.

Returning to the question of finding a desired profile, figure 7 shows how $d_{\mathrm{HZ}}, d_{\mathrm{SZ}}$ and their ratio vary with $\beta$ for all other parameters fixed. A multilammellar profile began to appear for $\beta<0.75$, while separation without a saturation zone appeared for $\beta>2.25$. Crucially, a bilayer-like profile could be found for all values of $\beta$ in the given range, meaning that the important ratio $d_{\mathrm{HZ}} / d_{\mathrm{SZ}}$ can be fine-tuned to the desired accuracy. See 3.3

\subsection{3 $\alpha$ and the effects of temperature}

Recalling the physical factors influencing $d_{\mathrm{SZ}}$ listed in 3.1 since $\alpha$ is inversely proportional to $T$, increasing $\alpha$ should increase the measurable $d_{\mathrm{Sz}}$. Because this can be seen in figure 3, it appears that temperature-related mechanisms can be captured in 1D. Indeed, turning to the incompressible free energy functional (2.7) in which $\alpha$ is scaled on $T$, increasing $\alpha$ (decreasing $T$ ) reduces the effect of the entropy relative to the interaction terms. This makes physical sense in that with less kinetic energy the hydrogen-bond network is more strongly preserved.

Further, the other physical temperature-related mechanism is that by which lower temperatures straighten the lipid tails on average. (Although the head groups also become "straighter" in the sense that they change position less and therefore have a longer averaged shape, the main effect is in the longer and more deformable tails.) As a result, we would expect the ratio $d_{\mathrm{HZ}} / d_{\mathrm{SZ}}$ of our model to decrease as $\alpha$ increases ( $T$ decreases), and this is seen in figure 4.

Finally, we caution against one potential method of setting $\alpha$. That increasing $\alpha$ (decreasing $T$ ) thickens the physical bilayer (in terms of $d_{\mathrm{SZ}}$ ) is from the 


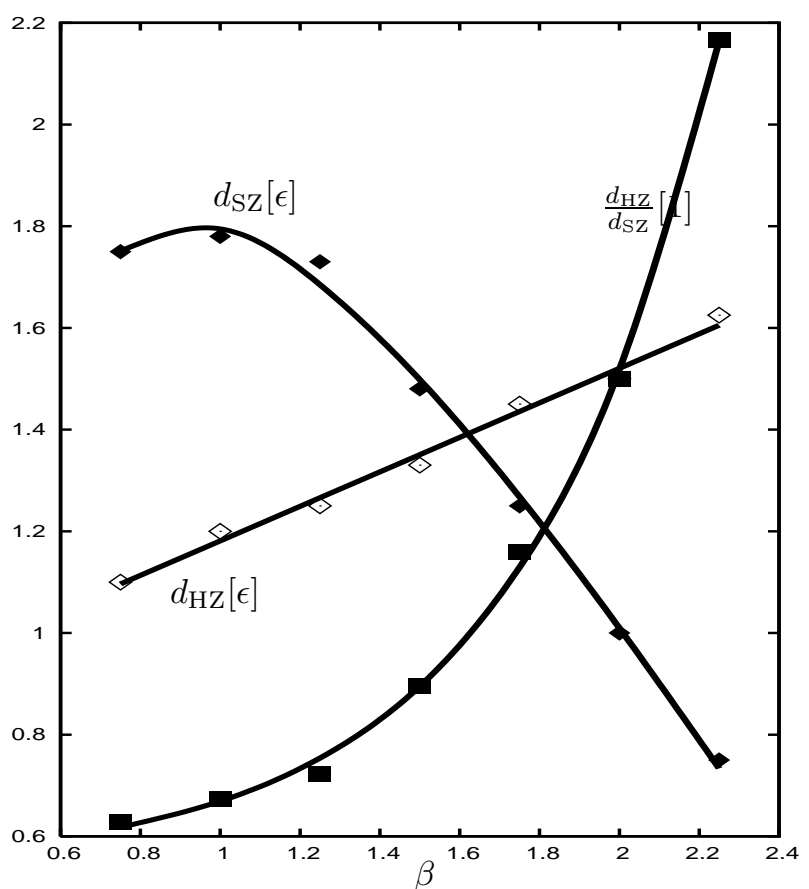

Figure 7: $d_{\mathrm{HZ}}, d_{\mathrm{SZ}}, d_{\mathrm{HZ}} / d_{\mathrm{SZ}}$ for different values of $\beta$. The other parameters were fixed: in particular, $\left(\alpha, \epsilon, c_{0}\right)=(3,2,0.024)$. For $d_{\mathrm{Hz}}$ the drawn line of best fit is straight $a x+c$, for $d_{\mathrm{SZ}}$ it is of the form $a x+b / x+c$, and for the ratio it is exponential and of the form $a \exp (b x)+c$.

view point of moving with the bilayer as it undergoes thermal fluctuations. But increasing $\alpha$ (decreasing $T$ ) should suppress these thermal fluctuations. If the model were capturing these undulations as well then we might expect $d_{\mathrm{SZ}}$ to decrease as $\alpha$ increased ( $T$ decreased) because the order of magnitude of the thermal fluctuations is larger than that of the changes in $d_{\mathrm{SZ}}$ (a few percent). Since we see the opposite, this precludes using the amplitude of an averaged thermal undulation to fix $\alpha$.

\subsection{Choosing a parameter set}

Based on $\S \$ 3.2 .113 .2 .213 .2 .3$, we can set the parameters to reproduce the profile of a desired lipid species bilayer whose properties are known a priori from experimental data as follows.

Choose $\alpha=3$ or 4 , noting from figure 4 that from the numerical viewpoint $\alpha$ controls the sensitivity of the ratio $d_{\mathrm{Hz}} / d_{\mathrm{SZ}}$ to variation in $c_{0}$. Then, recalling that the effects of $\epsilon$ and $\beta$ combine, we pick $\epsilon=2$ or 3, essentially only requiring clear separation of the head and tail regions. Then we set $\beta=1$ to start. Next, since $c_{0}, m$ combine with $m$ fixed and $c_{0}$ to vary, we run the numerics with a few $c_{0}$ until $d_{\mathrm{Hz}} / d_{\mathrm{SZ}}$ is close to the desired value. These results are then fine-tuned by varying $\beta$, which smoothly varies the values $d_{\mathrm{HZ}}$ and $d_{\mathrm{SZ}}$. 


\section{Conclusions}

We introduced a more physical model of the hydrophobic effect into the continuum paradigm of Blom and Peletier (2004) and showed that one-dimensional numerical solutions can reproduce key characteristics of physical lipid bilayers. The realised goal was to show that a more physical model enabling better connections with microscale numerics and first principles is capable within the paradigm of generating physically realistic bilayer density profiles. In particular, the mechanically-important bilayer thickness can be reproduced. Indeed, various key characteristics were shown to behave physically, at least one in contrast to the original model. The coarse-graining or smoothing of the molecular information inherent in the paradigm was clarified, and the smoothed numerical data calibrated to measurements of physical bilayers. Examining some of the key parameters in turn, we gave a strategy for setting their values, noting that future work, especially in higher dimensions, could make this process even more robust by further appeal to physical arguments. In particular, $\alpha$ is formally linked to (the inverse of) the temperature, and has been shown here to have that effect on the numerical results, while $\beta$ should be related to the range of the hydrogen bonding forces. Already, however, the new parameter $\beta$ has been seen here to introduce the short-range interaction tool, allowing analytical results to be obtained with greater ease.

Much work remains to show that the BP paradigm is a viable mesoscale tool for multiscale simulations. The main aim is to work in higher dimensions, and include compressibility effects by allowing $p$ to vary. The former represents a significant computational challenge. If the conclusions of the current paper are supported by higher-dimensional work, then the paradigm can be made as physical as desired by varying $\gamma$, considering other $\kappa$ (and indeed whether "promoting proximity" is the best model of the underlying physics), including electrostatic effects, weak head-head van der Waals repulsion terms, and so on. The level of detail will rest on computational issues, in particular cost-benefit considerations in the light of the paradigm's use as a mesoscale numerical filter.

\section{Acknowledgements}

P.L.W. gratefully acknowledges the support of the Japan Society for the Promotion of Science and the University of Tokyo's Intelligent Modeling Laboratory during part of this work. His thanks for many helpful discussions go to members of the IKEMEN group of the Fluid Engineering Laboratory of the University of Tokyo. We also thank the RIKEN institute for their support through the project Research Program for Computational Science, R\&D Group for the Next-generation Integrated Simulation of Living Matters, which is supported by the Japanese Ministry of Education, Culture, Sports, Science and Technology.

\section{References}

Ayton, G., Smondryev, A. M., Bardenhagen, S. G., McMurty, P., and Voth, G. A. (2002). Calculating the bulk modulus for a lipid bilayer with nonequilibrium molecular dynamics simulation. Biophys. J., 82:1226-1238. 
Blom, J. G. and Peletier, M. A. (2004). A continuum model of lipid bilayers. European J. Appl. Math., 15:487-508.

Boal, D. (2002). Mechanics of the Cell. CUP, Cambridge.

Boryczko, K., Dzwinel, W., and Yuen, D. A. (2003). Dynamical clustering of red blood cells in capillary vessels. J. Mol. Model., 9:16-33.

Chandler, D. (2005). Interfaces and the driving force of hydrophobic assembly. Nature, 437:640-647.

Fraaije, J. G. E. M. (1993). Dynamic density functional theory for microphase separation kinetics of block coploymer melts. J. Chem. Phys., 99:9202-9212.

Immergut, E. H., editor (1991). Encyclopedia of Applied Physics, volume 18. $\mathrm{VCH}$, Berlin.

Kronberg, B., Costas, M., and Silveston, R. (1995). Thermodynamics of the hydrophobic effect in surfactant solutions - micellization and adsorption. Pure \& Appl. Chem., 67:897-902.

Lee, A. G. (2003). Lipid-protein interactions in biological membranes: a structural perspective. Biochim. Biophys. Acta, 1612:1-40.

Mchedlishvili, G. and Maeda, N. (2001). Blood flow structure related to red cell flow: determination of blood fluidity in narrow microvessels. Jpn J. Physiol., 51:19-30.

Mouritsen, O. (2005). Life - as a Matter of Fat. Springer, Berlin.

Sugii, T., Takagi, S., and Matsumoto, Y. (2005). A molecular-dynamics study of lipid bilayers: effects of the hydrocarbon chain length on permeability. $J$. Chem. Phys., 123:184714.

Sugii, T., Takagi, S., and Matsumoto, Y. (2007). A meso-scale analysis of lipid bilayers with the dissipative particle dynamics method: thermally fluctuating interfaces. Internat. J. Numer. Methods Fluids, 54:831-840.

Vaidya, N. K., Huang, H., and Takagi, S. (2007). Modelling HA proteinmediated interaction between an influenza virus and a healthy cell: pre-fusion membrane deformation. Math. Med. Biol., 24:251-270.

Wiener, M. C. and White, S. (1992). Structure of a fluid dioleoylphophatidylcholine bilayer determined by joint refinement of x-ray and neutron diffraction data. Biophys. J, 61:434-447.

Young, D. C. (2001). Computational Chemistry: A Practical Guide for Applying Techniques to Real-World Problems. John Wiley \& Sons, Inc., New York. 